\title{
IMAGE
}

\section{Simultaneous corrective osteotomy and fracture fixation in a patient with polydactyly}

Young Ho Kim, Ja Hea Gu

Department of Plastic Surgery, Dankook University Hospital, Cheonan, Korea

Correspondence: Ja Hea Gu

Department of Plastic Surgery, Dankook University Hospital, 201 Manghyangro, Dongnam-gu, Cheonan 31116, Korea

Tel: +82-41-550-3873, Fax: +82-41-554-6477

E-mail: jaheagu@gmail.com

This article was presented as a poster at the FESSH XXII on June 21-24, 2017, in Budapest, Hungary.

Received: July 26, 2018 • Revised: December 27, 2018

- Accepted: May 30, 2019

pISSN: 2234-6163・ elSSN: 2234-6171

https://doi.org/10.5999/aps.2018.01053

Arch Plast Surg 2019;46:610-611

Copyright (c) 2019 The Korean Society of Plastic and Reconstructive Surgeon

This is an Open Access article distributed under the terms of the Creative Commons

Attribution Non-Commercial License (http://creativecommons.org/licenses/by-nc/4.0)

which permits unrestricted non-commercial use, distribution, and reproduction in any

medium, provided the original work is properly cited.

The reoperation rates for deformity correction after corrective surgery for polydactyly have been reported to be between $12 \%$ and $26 \%[1,2]$. In our literature review, no reports were found of a simultaneous secondary revision operation and fracture fixation in a polydactyly patient after trauma. We report the treatment of a 13-year-old boy via simultaneous secondary corrective osteotomy, first web space release, scar release, and fracture fixation, with an acceptable result.

A 13-year-old patient visited our clinic presenting of a swollen and painful thumb after trauma.

Computed tomography and an X-ray examination were performed, followed by a diagnosis of fracture in the left thumb proximal bone and subluxation of the metacarpophalangeal joint of the left thumb (Fig. 1) We found that the patient had been treated for his duplicate thumb at 1 year of age. He later suffered from a deformity and limited motion, but had not visited a clinic (Fig. 2). The patient and his guardian wanted to correct these problems simultaneously if possible. We planned an open reduction with percutaneous K-wire pinning and corrective wedge osteotomy, tendon realignment, and scar release with multiple Z-plasty and a four-flap operation. Intraoperatively, we found that the patient had undergone excision of the extra radial-side thumb

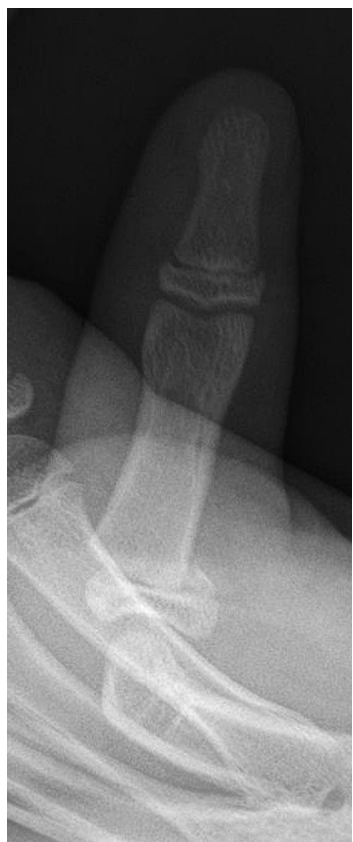

Fig. 1.

Preoperative X-ray view. A simple anteroposterior $\mathrm{X}$-ray of the thumb revealed a fracture in the proximal metaphysis with physeal separation of the left thumb metacarpal bone (Salter-Harris type II). In addition, subluxation of the metacarpophalangeal joint of the left thumb was observed.

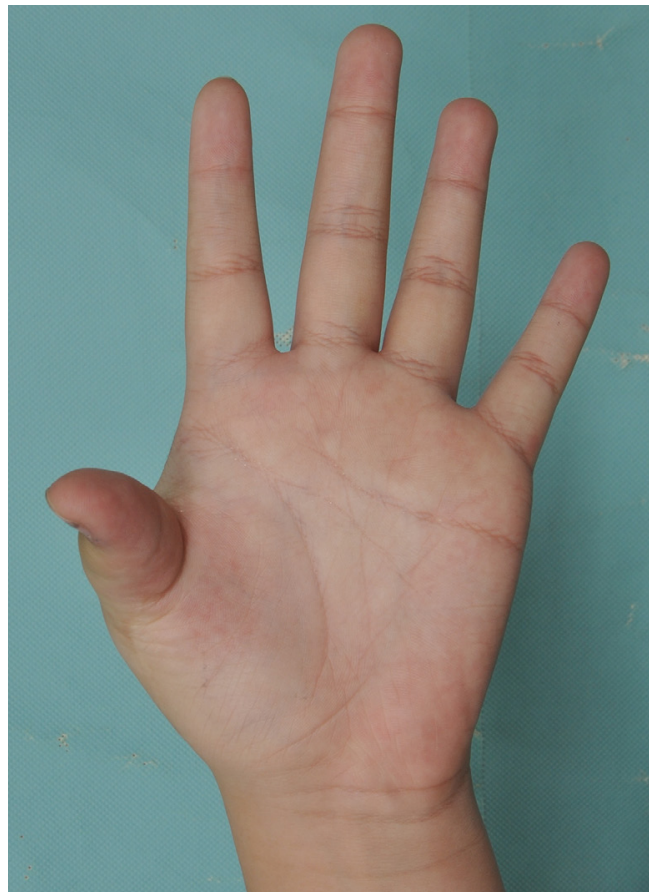

Fig. 2.

Preoperative photograph. Radial deviation, unstable metacarpophalangeal abduction, first web space narrowing, and scar contracture were found after polydactyly reconstruction. The patient showed limited abduction and extension of the metacarpophalangeal joint of the left thumb.

and reconstruction of the radial collateral ligament at the proximal metacarpal head, which remained attached to the ulnar-side thumb by Ethibond; however, the ligament was stretched. After reduction 

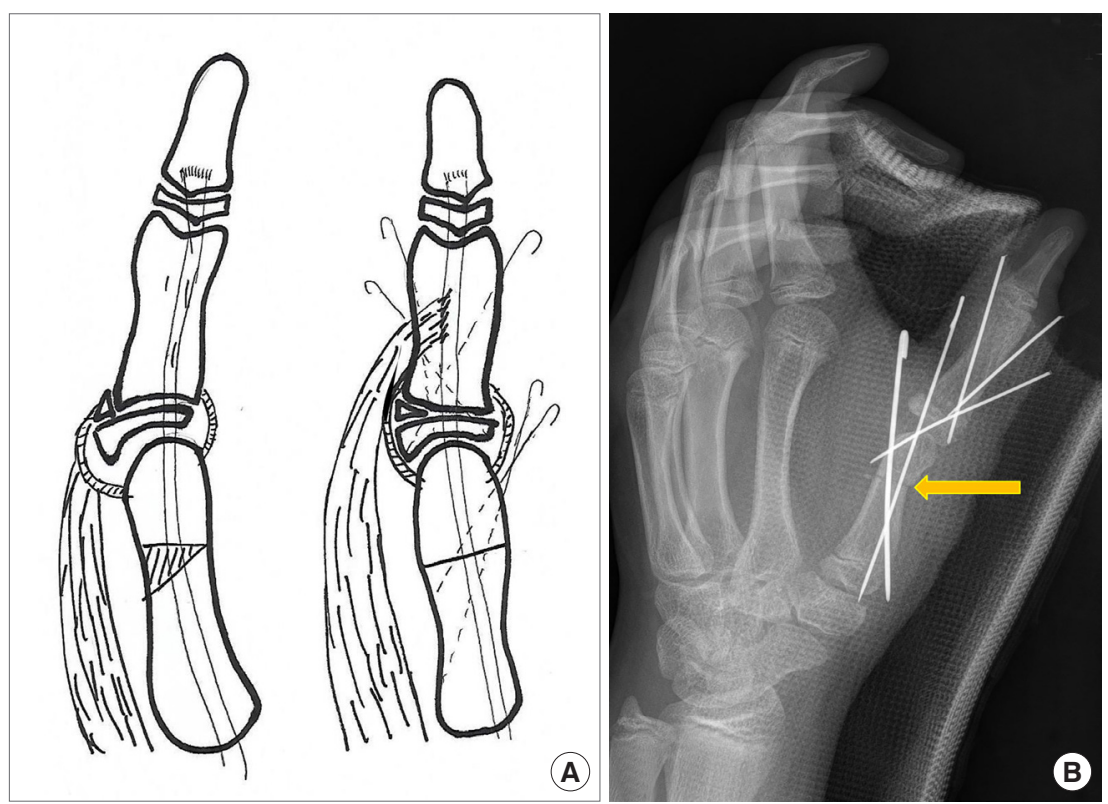

Fig. 3.

Illustration of the operative plan (A) and immediate postoperative X-ray (B). (A) Wedge osteotomy $\left(30^{\circ}\right)$ was planned for the metacarpal bone. We treated the fracture of the proximal phalanx with open reduction and percutaneous pinning with a K-wire according to our usual protocol. Next, the collateral ligament was reinforced and the abductor pollicis brevis muscle was resuspended onto the thumb. (B) We treated the polydactyly deformity with corrective wedge osteotomy (yellow arrow) and tendon realignment. of the fractured proximal bone, osteotomy was done on the metacarpal bone to correct the alignment by about $30^{\circ}$. The alignment of the proximal phalanx was corrected not by osteotomy, but by fractured site rearrangement. Then, the collateral ligament was reinforced and the abductor pollicis brevis muscle was resuspended onto the thumb (Fig. 3).

At 10 weeks postoperatively, the patient and his parents were satisfied with the shape of his thumb and its improved motion, while the fracture site also showed good healing (Supplemental Fig. 1). A simultaneous secondary revision operation can thus be considered in polydactyly patients who experience trauma, such as fracture or dislocation.

\section{Notes}

\section{Conflict of interest}

No potential conflict of interest relevant to this article was reported.

\section{Ethical approval}

The study was performed in accordance with the principles of the Declaration of Helsinki. Written informed consent was obtained.

\section{Patient consent}

The patient provided written informed consent for the publication and the use of his images.
Author contribution

Writing original draft, data collection, reference review: Kim YH. Review, Final editing, approval of final manuscript: $\mathrm{Gu} \mathrm{JH}$.

\section{ORCID}

Young Ho Kim https://orcid.org/0000-0001-69149637

Ja Hea Gu https://orcid.org/0000-0002-4672-2856

Supplementary material

Supplemental Fig. 1. Photograph and simple X-ray at 10 weeks postoperatively. (A) A simple X-ray demonstrated healing of the fracture and osteotomy site. (B) Improvements were observed in metacarpophalangeal abduction stability and extension mobility of the metacarpophalangeal joint of the left thumb.

Supplemental data can be found at: https://doi.org/10.5999/aps.2018.01053.s001

\section{References}

1. Stutz C, Mills J, Wheeler L, et al. Long-term outcomes following radial polydactyly reconstruction.J Hand Surg Am 2014;39:1549-52.

2. Ganley TJ, Lubahn JD. Radial polydactyly: an outcome study. Ann Plast Surg 1995;35:86-9. 